\title{
Federico Pascual del Roncal (1903-1958), psicólogo y traductor ${ }^{1}$
}

\author{
Ramón León \\ Universidad Ricardo Palma \\ Lima, Perú
}

Recibido: 22 de julio de 2015 / Aprobado: 8 de agosto de 2015

Federico Pascual del Roncal (1903-1958) fue un psiquiatra español, quien obligado a abandonar España tras el ascenso al poder de Franco, emigró a México, donde de modo simultáneo con su práctica profesional ejerció la docencia en la Universidad Nacional Autónoma. Además, publicó un libro dedicado a la psiquiatría infantil (Manual de neuropsiquiatría infantil; 1940, 1952) y otro al psicodiagnóstico de Rorschach (Técnica del psicodiagnóstico de Rorschach, 1949), y llevó a cabo la traducción al castellano de dos importantes libros del psicólogo alemán Werner Wolff y del estudio sobre conducta sexual en el varón, de Alfred C. Kinsey.

\section{Federico Pascual del Roncal / psicólogo / México}

\section{Federico Pascual del Roncal (1903-1958), Psychologist and Translator}

This article is a biographical sketch of Federico Pascual del Roncal (1903-1958), a Spanish psychiatrist of leftist political ideology, who after the Spanish Civil War, left his country for Mexico. There he started a new carrier as a lecturer at the Universidad Nacional Autónoma de México and wrote a number of papers on psychiatric and neurological topics and two books on Rorschach and child psychiatry. Furthermore, Pascual del Roncal translated into Spanish two books of Werner Wolff, a German psychologist exiled in USA, an important book by Lemkau about mental hygiene, and a book on sexual behavior in male by Kinsey.

\section{Federico Pascual del Roncal / psychologist / Mexico}

\footnotetext{
1 El autor expresa su agradecimiento al doctor Miguel Marco Igual (Barcelona, España) por su valiosa ayuda en la preparación de la parte biográfica de Federico Pascual del Roncal, especialmente de sus años españoles. Este trabajo fue realizado gracias al apoyo de la Fundación Alexander von Humboldt, de la República Federal de Alemania.

Correo electrónico: Rld310850@yahoo.com.mx
} 
A pesar de su importancia en la difusión del conocimiento científico (Montgomery, 2000), la historia de la traducción científica ha sido apenas tratada (Olohan y Salama-Carr, 2011). Solo desde fecha relativamente reciente su estudio es considerado un tema de interés por derecho propio (Rupke, 2000; Petrou, 2006); en los últimos años, Shuping (2013) ha tratado aspectos lingüísticos de ella, en tanto que Gordin (2012) se ha referido a la traducción aplicada a la química, así como en el contexto de la Guerra Fría. Montgomery (2009), por su parte, se ha preguntado si el declinamiento de Estados Unidos como primera potencia mundial afectará el prestigio del inglés como el idioma de comunicación internacional. Lee-Jahnke (2005), a su vez, se interroga acerca de las características de un traductor médico competente. El libro The Routledge Handbook of Translation Studies de Millan y Bartrina (2013) ofrece una visión panorámica muy interesante de los problemas de la traducción en los últimos tiempos.

Las situaciones políticas y económicas tienen un papel significativo, por ejemplo, en las últimas décadas la traducción árabe-inglés (y viceversa) ha cobrado gran importancia; asimismo, el idioma chino, hasta no hace mucho poco estudiado como segunda lengua, es hoy enseñado en numerosas universidades latinoamericanas, dado el activo intercambio comercial entre China y América Latina. Por otro lado, el castellano es cada vez más hablado en Estados Uni- dos debido al elevado porcentaje de la población de origen latinoamericano.

Surgida la psicología en el mundo anglosajón, las obras de los grandes psicólogos europeos y norteamericanos se han difundido en el mundo iberoamericano gracias al trabajo de los traductores. Quintana, Rosa, Huertas y Blanco (1997) han pasado revista a siete décadas de traducciones. Para solo mencionar un ejemplo, sin duda el más conocido, los lectores del mundo de habla castellana accedieron a la obra de Sigmund Freud gracias al trabajo de Luis López-Ballesteros y de Torres (18961938), nombre vinculado definitivamente al psicoanálisis.

En el presente artículo nos referimos a un psiquiatra español exiliado en México, que aparte de su obra propia (hoy lamentablemente olvidada), también llevó a cabo un importante trabajo de traductor: Federico Pascual del Roncal (1903-1958).

\section{El CONTEXTO SOCIAL}

La Guerra Civil española dio lugar a la emigración de un gran número de intelectuales y científicos españoles opuestos al régimen de Francisco Franco. No hay cálculos precisos acerca de la cantidad de los emigrantes, pero sí se está de acuerdo en que la partida de ellos supuso una grave e irreparable pérdida de sustancia para la vida cultural y científica de España: médicos, juristas, filósofos, psicólogos, escritores, pensadores, 
artistas, dejaron su patria (Gracia, 2010). Fue América Latina la región que probablemente recibió el mayor número de emigrantes debido a los lazos culturales y a la ausencia de barreras idiomáticas. México, por ejemplo, acogió alrededor de 25000 inmigrantes españoles, de los cuales un $25 \%$ estaba constituido por científicos e intelectuales (León, 2006), "la flor y nata de la intelectualidad española del primer tercio del siglo XX" (Marco, 2010, p. 24).

Los años iniciales de estos emigrados en el Nuevo Continente fueron muy duros. Solo unos pocos, como August Pí y Sunyer (1874-1965), reconocido fisiólogo catalán, llegaron precedidos de un gran prestigio, y eso les permitió alcanzar posiciones académicas elevadas y rehacer prontamente su vida.

No fue ese, sin embargo, el caso de la gran mayoría, que debió enfrentarse a una serie de dificultades. Un caso ilustrativo es el de Emilio Mira y López, quien al momento de la emigración era una personalidad ampliamente conocida en la psiquiatría y la psicología europeas, no obstante lo cual fue activamente difamado en su país (González, 2009). Tras su emigración a Argentina, Mira y López tuvo que contentarse con "coleccionar ofertas de trabajo" (Mira, 1941), sin que ninguna de ellas se concretara, para finalmente instalarse en Brasil, país en el cual se le ofreció una posición académica acorde con sus merecimientos.
Poseedores de una formación sólida y en muchos casos con el conocimiento de varios idiomas, algunos de los emigrantes, al comienzo de su estancia en esta parte del mundo, encontraron en la traducción una forma de subsistir mientras se hacían a la búsqueda de una posición profesional estable y adecuadamente remunerada. Ese fue el caso del ya mencionado Mira y López (León y Kagelmann, 1992).

Otros intelectuales emigrados, sobre todo filósofos y juristas, a la par con sus tareas académicas realizaron labores de traducción de manera casi permanente.

\section{Biografía}

Federico Pascual del Roncal nació en Zaragoza, el 13 de febrero de 1903. Atraído por la medicina, llevó a cabo los estudios correspondientes en la Universidad de Santiago de Compostela, recibiéndose en 1930 y continuando estudios doctorales en la Universidad de Madrid (Marco, 2010). Optó por la especialidad de psiquiatría infantil.

Completó su formación en Praga, Checoslovaquia, gracias a una beca de la Junta de Ampliación de Estudios (JAE). En esa ciudad se formó en las clínicas neurológica y psiquiátrica, y -siempre según Marco Igual (2010)- fue también profesor en el Instituto Iberoamericano de la Universidad de Praga.

En ese país, de acuerdo con la información que da a conocer Páramo 
Ortega (1992), Pascual del Roncal se capacitó al lado del neurólogo Ladislav Haskovec (1866-1944), destacada figura de la especialidad en su país.

En el ámbito político, fue integrante del Partido Comunista Español. Desempeñó hacia 1936 la jefatura del Departamento de Psiquiatría e Higiene Mental del Consejo Superior Psiquiátrico Ministerio de Sanidad (Cerón, 1996) y fue médico de varias instituciones psiquiátricas en la urbe madrileña, por ejemplo el Hospital de Ciempozuelos y en la Clínica Psiquiátrica de Rodríguez Lafora, su maestro, en el Hospital General de Madrid.

Asimismo, fue asistente del Instituto del Cerebro de Moscú. En 1935 le fue concedido el premio Röel (accésit) de la Sociedad Española de Higiene por el tema "El silencio es el primer deber del ciudadano" $(A B C, 17$ de febrero de 1936). Llevó a cabo trabajos sobre el fósforo sanguíneo en las psicosis. De acuerdo con López y León (2001), había establecido contacto con el psicoanálisis durante su estancia en Checoslovaquia.

Pascual del Roncal era un hombre de amplia cultura, sustentada en el conocimiento de varias lenguas: inglés, francés, alemán, ruso y checo.

De acuerdo con Marco Igual (2010), al inicio de la Guerra Civil pidió sin éxito incorporarse al servicio diplomático de la República en la Legación Española de Praga.
En 1937 lo encontramos en Valencia, convertida tras la derrota de la Segunda República y el establecimiento de la dictadura de Franco en la capital de la República y el más importante centro intelectual de España (Álvarez y Huertas, 1987), donde tuvo un papel importante en la organización de la Casa de la Cultura, fue secretario de la revista Madrid. Cuadernos de la Casa de la Cultura (Marco, 2010).

De acuerdo con Marco (2010), en mayo de 1938 se incorporó a la Embajada española en Moscú, en calidad de segundo secretario y colaborador del primer secretario Vicente Polo, quien debido al traslado de Marcelino Pascua (hasta ese momento embajador español) a París, era el más alto funcionario de la embajada.

En 1939 se vio obligado a emigrar a México, formando parte del numeroso contingente de estudiosos españoles de la conducta que emprendieron el camino del exilio, entre quienes también estaban Gonzalo Rodríguez Lafora, Dionisio Nieto (1905-1987) y José Peinado Altable (1909-1995). Ya en México difundiría la doctrina de Freud, y se ha llegado a afirmar que ejercía como psicoanalista sin haber sido psicoanalizado (Capetillo, 2010).

Tras su arribo a ese país fue acogido por La Casa de España, entre 1939 y 1948 (Barona, 2003). En 1940 lo encontramos como profesor de la Universidad Nacional Autónoma de México (UNAM), en la que su labor en la en- 
señanza de pruebas proyectivas ha sido destacada (Hernández, 2007). En 1943 se desempeñó en la Secretaría de Educación y en 1943 fue nombrado subdirector de la Clínica Neuro-psiquiátrica Falcón (Amo y Shelby, 1950), cargo que mantuvo hasta su muerte, en 1958.

Miembro de la Sociedad Mexicana de Eugenesia, Pascual del Roncal formó parte de la junta directiva de esta institución en el periodo 1948-1949, en calidad de secretario de actas, y con la presidencia de Feliciano Sánchez Ríos (Gaceta Médica de México, 31 de diciembre de 1948). En 1948 aparece como responsable de las asignaturas de Psicodiagnóstico de Rorschach y de Higiene Mental y Psicoterapia en el programa de Maestría en Psicología de la UNAM. Asiduo y activo participante en congresos, se registra su asistencia al I y II congresos interamericanos de Psicología, celebrado en la ciudad de México en 1954; en ese mismo año asiste al Primer Congreso Nacional de Psicología, en Tucumán, Argentina. Asimismo, presentó una ponencia sobre el test de Rorschach en esquizofrénicos en el I Congreso Latinoamericano de Psicología celebrado en Montevideo en 1950 (León, 1981).

A fines de la década de 1940 el psicoanálisis era una rama que despertaba mucho interés en México, lo que fue más estimulado aún por la presencia de Erich Fromm (1900-1980), quien permaneció en ese país entre 1949 y 1973 (Derbez, 1981; Millán y Gojman, 2000). En ese entonces, cuatro médicos intere- sados en la obra de Freud organizaron un curso en el Pabellón de ObservaciónHombres del manicomio La Castañeda. Esos médicos fueron Aniceto Aramoni (1916-2012), Arturo Higareda, Armando Hinojosa y Jorge Derbez, que coordinaron con Raúl González Enríquez. En el curso colaboraron, al lado de Pascual del Roncal (dedicado a las pruebas psicológicas), Guillermo Dávila (psicopatólogo), José Luis Patiño y Abraham Fortes (clínicos); Dionisio Nieto (neuropatólogo) y los antropólogos Monzón y Esteva (Varelay Vásquez, 2014).

La producción científica de Pascual del Roncal fue considerable. Trabajos suyos fueron publicados en los Archivos de Neurología y Psiquiatría de México (Obrador, Pascual del Roncal y Falcón, 1943; Pascual del Roncal, 1939a, 1945), así como en la Revista Médica del Ateneo Ramón y Cajal (Pascual del Roncal, 1947a; 1947b), una institución fundada en 1949, que agrupaba a académicos españoles exiliados en México (López, 2009). También la Revista de Medicina y Ciencias Afines acogió un trabajo de su autoría dedicado a la gerontología (Pascual del Roncal, 1951). Asimismo, investigaciones y ensayos escritos por el psiquiatra español aparecieron también en revistas mexicanas dedicadas a temas forenses (Pascual del Roncal, 1940a; 1941a) y en medios especializados del extranjero (Pascual del Roncal, 1939b; 1953; 1954). Ocasionalmente publicó comentarios de libros (Pascual del Roncal, 1944; 1947c), y recopiló materiales para la obra de otro autor. 
En 1940 apareció su Manual de neuropsiquiatría infantil (Pascual del Roncal, 1940b), que en su momento tuvo una amplia difusión, dando lugar a una segunda edición en 1952, en La Prensa Médica Mexicana. Nueve años más tarde dio a la luz Teoría y práctica del psicodiagnóstico de Rorschach (Pascual del Roncal, 1949).

En la obra escrita de Pascual del Roncal es posible distinguir varias líneas de trabajo, relacionadas con diferentes etapas de su vida.

En primer lugar, hay escritos referidos a aspectos neurológicos y neuroquímicos, un área en la cual había aportado ya en su país natal, y que él cultivó también durante la etapa mexicana de su existencia. En esto se puede reconocer la influencia de la prestigiosa tradición de estudios del sistema nervioso establecida por Santiago Ramón y Cajal (1852-1934), Premio Nobel de Medicina en 1906, y proseguida por sus discípulos, como Gonzalo Rodríguez Lafora, con quien Pascual del Roncal trabajara.

Por otro lado, encontramos varios estudios dedicados a tratar la aplicación de la lobotomía, una técnica desarrollada por el médico portugués Egas Moniz (1874-1955), y que se difundiera rápidamente por el Nuevo Mundo, tanto de habla inglesa como hispanohablante (El-Hay, 2007).

La neuropsiquiatría es, asimismo, otra línea de trabajo a la cual Pascual del Roncal dedicó mucha energía. La evidencia más clara nos la ofrece su Manual de neuropsiquiatría infantil (Pascual del Roncal, 1940b). Algunos temas tratados por nuestro autor reflejan denominaciones y planteamientos de la época, como no podía ser de otro modo; así, dedica un trabajo a la demencia precocísima, denominación en boga para lo que después se denominaría esquizofrenia infantil.

Cabe mencionarse, asimismo, sus trabajos dedicados a temas criminológicos y eugenésicos. No debe olvidarse que Pascual del Roncal era miembro de la Sociedad Mexicana de Eugenesia.

Por último, está la línea de trabajo que gira en torno a la evaluación psicológica, cuyo principal testimonio es su libro dedicado al psicodiagnóstico de Rorschach, probablemente uno de los primeros provenientes de la pluma de un especialista residente en América Latina.

Esta obra suya continúa siendo citada, en tanto que otros libros latinoamericanos sobre el particular, en especial el de Julio Endara (1898-1969), importante psiquiatra ecuatoriano, está hoy olvidado (Endara, 1964).

\section{La labor de traducción de Pascual DEL RONCAL}

Debemos destacar, por otro lado, el importante trabajo cumplido por Pascual del Roncal como traductor: es posible registrar cuatro obras traducidas al castellano por él. 
La primera obra que tradujo fue Sexual behavior in male, el importante libro de Alfred C. Kinsey, aparecido originalmente en 1948 (Kinsey, Pomeroy y Martin, 1948). Tan solo un año después, la editorial Interamericana ponía a la disposición de los lectores latinoamericanos una versión castellana de este título llevada a cabo por Pascual del Roncal (Kinsey et al., 1949).

No es posible precisar cómo fue que Pascual del Roncal se hizo cargo de esta traducción. Las razones por las cuales las llevó a cabo no requieren de mayor indagación: la aparición del informe de Kinsey encontró pronta recepción y generó numerosos comentarios (por cierto, no todos favorables) en la prensa especializada norteamericana. Más allá de las controversias a las que dio lugar, era evidente que se trataba de una obra de gran importancia.

Es probable que la presencia del nombre de Pascual del Roncal asociado a la versión castellana del informe Kinsey lo colocara en la categoría de potenciales traductores de otras obras del área de la psicología y de la psiquiatría. Esta suposición nos parece correcta, dado que poco tiempo después llevaría a cabo la traducción de dos libros de Werner Wolff (1904-1957), psicólogo alemán que emigrara a Estados Unidos y que desempeñara un rol importante en la constitución de la Sociedad Interamericana de Psicología, así como en los primeros congresos de esa institución (Colotla y Urra, 2006; León, 2012).
Wolff, autor prolífico a pesar de su prematura muerte, dio a la luz en inglés dos breves y muy didácticas introducciones, una a la psicología (1947) y otra a la psicopatología (1950), las mismas que fueron traducidas por Pascual del Roncal por encargo del Fondo de Cultura Económica, con los títulos de Introducción a la psicología (Wolff, 1953) e Introducción a la psicopatología (Wolff, 1956).

Se trata de dos obras que en castellano han hecho una carrera excelente, si se tiene en cuenta el número de reimpresiones de cada una de ellas. Aun hoy, a pesar de los muchos años transcurridos desde su aparición, estos dos libros de Wolff siguen siendo consultados.

Casi al mismo tiempo después de la traducción de los libros de Wolff, Pascual del Roncal llevó a cabo en 1953 la traducción de Mental Hygiene (1949), una obra de Paul V. Lemkau (19091992), uno de los pioneros del movimiento de higiene mental. Esta obra ha alcanzado también varias reediciones en nuestro idioma.

\section{Comentario}

El trabajo del traductor, no solo del de obras literarias sino también del que realiza traducciones científicas, es ingrato. Cuando tiene una obra en la mano, por lo general el lector no se preocupa en averiguar quién es el que la ha vertido en el idioma en el que habrá de leerla. Eso ocurre también en el caso de la psicología y de la psiquiatría. 
Sin embargo, lo cierto es que muchos psiquiatras y psicólogos destacados han llevado a cabo trabajos de traducción. Limitándonos solo al mundo de habla hispana debemos recordar que el peruano Honorio Delgado (1892-1969), introductor del psicoanálisis en el Nuevo Mundo, tradujo al castellano (en colaboración con Paul Wilson) una obra de Smith Elly Jelliffe (1866-1945), importante seguidor norteamericano de las ideas de Sigmund Freud (Jelliffe, 1929).

Mira y López, por su parte, vertió al castellano el Tratado de psicoterapia de Paul Schilder (1886-1941), importante psiquiatra alemán obligado a emigrar a Estados Unidos (Schilder, 1945).

Es probable que también fuera en los años de su estancia en Argentina cuando tradujera y prologara un libro de Frolov (1965), que trata de la teoría de Pavlov. Florencio Villa Landa (1912-1992), un psiquiatra español radicado primero en la Unión Soviética y después en Cuba, llevó a cabo importantes traducciones de libros rusos de psicología al castellano, como lo acreditan Psicología (Smirnov, 1960) y Psiquiatría (Sluchevski, 1960). Asimismo, Rubén Ardila tradujo al castellano dos obras de gran importancia: Verbal behavior, de B. F. Skinner (1957, 1981), y la de Edwin G. Boring, A history of experimental psychology (Boring, 1950), un verdadero clásico en el campo de la historia de la psicología (Boring, 1988).
Las motivaciones por las cuales las personas emprenden el trabajo de traductor son, obviamente, de lo más variadas. Al lado de la intrínseca, vinculada al deseo de dar a conocer un trabajo que es considerado valioso, debe también señalarse que el trabajo de traductor ofrece la posibilidad de generar ingresos.

Entre los emigrantes españoles en México encontramos a quienes, en sus primeros años en el exilio, optaron por llevar a cabo traducciones. La activa labor de numerosas y prestigiadas editoriales mexicanas jugó un papel no menos importante. Pero no solo en México: ya hemos señalado el caso de Emilio Mira y López, establecido por un tiempo en Argentina, otro país con un fuerte movimiento editorial, quien, en su correspondencia con Walter Blumenfeld le indica que está llevando a cabo algunas traducciones mientras espera una posición profesional en Argentina que nunca habría de llegar (León y Kagelmann, 1992).

Pascual del Roncal se encontraba en una posición apropiada para llevar a cabo también labores de traducción. Políglota y con amplios intereses culturales, profesor en la prestigiosa Universidad Nacional Autónoma de México, era un profesional de interés para una editorial como el Fondo de Cultura Económica, que desplegó desde sus inicios una muy activa y hasta hoy ininterrumpida labor de dar a conocer clásicos de las más diversas ramas del 
saber en versiones castellanas, realizadas con gran rigor y cuidado.

Pero no debemos destacar solo el trabajo de traductor cumplido por este exiliado español en detrimento de su obra propia. Su Manual de neuro-psiquiatría infantil constituyó un valioso aporte a la literatura latinoamericana especializada sobre el tema, si bien se puede observar un desbalance en el tratamiento a favor de las consideraciones psiquiátricas sobre las neurológicas. Con Pascual del Roncal se inicia también en 1945 el estudio del Rorschach en México.

Con respecto a su participación en el movimiento eugenésico en México, debemos señalar que la palabra eugenesia tiene hoy una connotación negativa y está vinculada de modo abierto a prácticas racistas (Kevles, 1995). Sin embargo, no siempre fue así. A comienzos del siglo XX la eugenesia era considerada una disciplina seria, tanto que importantes figuras de la cultura y de la ciencia europea se encontraban entre sus seguidores. Ya hemos mencionado el hecho de que Ladislav Haskovec, el que fuera profesor de Pascual del Roncal durante sus años en Checoslovaquia, formaba parte activa del movimiento eugenésico de su país.

También en Estados Unidos había una actitud favorable hacia la eugenesia, inclusive en los círculos de la política. Combinando la teoría darwinista de la evolución con el conocimiento que venía proporcionando la genética, quienes promocionaban la eugenesia tenían la esperanza de desaparecer muchos desórdenes (tales como el retardo mental) en una generación:

Ellos entendían el comportamiento humano en simples términos genéticos: características tales como la inteligencia, la honestidad y la sobriedad eran transmitidos según la genética mendeliana. El futuro, por tanto, estribaba en promover el matrimonio de parejas "apropiadas" (fit) y desaconsejar la paternidad de otras. Dado que las personas con retardo mental no podían entender la sabiduría de la eugenesia, muchos estados emitieron leyes que permitían la esterilización involuntaria de adultos con retardo mental. (Brosco, Mattingly y Sanders, 2006, p. 303)

Pascual del Roncal fue miembro de la Sociedad de Eugenesia de México y publicó un artículo en la revista de dicha organización, con resonancias de la teoría de Lamarck (Pascual del Roncal, 1941b). Sorprende, ciertamente, que un hombre proveniente de la izquierda mostrara interés por la doctrina eugenésica, pero ese interés debe ser entendido en el contexto de su época. Sobre el particular, Suárez y López Guazo (2000) escribe:

En México, el panorama general de las ciencias humanas -antropología, sociología, medicina legal, biología humana- desde el inicio de los treinta hasta finales de los sesenta, estaba cargado de concepciones hereditaristas. La idea lombrosiana del criminal nato, la noción gal- 
toniana de la herencia del talento e inteligencia, así como la tesis de la degeneración, la tendencia a la transmisión del pauperismo, de diversas toxicomanías, la predisposición a la prostitución, e incluso la innata condición antisocial de los homosexuales. La preocupación del gremio médico y los penalistas, por la reproducción de los caracteres socialmente indeseables, se encuentra prácticamente en todas las publicaciones de la época. (p. 698)

Algo semejante sucede con su interés por la lobotomía. Hoy criticada y considerada como un procedimiento prohibido, la lobotomía fue ampliamente empleada en los años treinta y cuarenta no solo en Estados Unidos, sino también en Europa y en América Latina. Así, en la Gaceta Médica de México puede encontrarse un trabajo de Manuel Guevara Oropeza (1943) dedicado a la lobotomía prefrontal como tratamiento psiquiátrico. En Brasil, Aloysio de Mattos Pimenta (1913-1987) llevó a cabo la primera intervención de esta naturaleza en 1936 (Pimenta, 1936), habiéndose reportado aplicaciones en masa (Masiero, 2003). En el Perú, J. Óscar Trelles (1904-1990), distinguido neurólogo formado en Francia, publica en la Revista de Neuro-psiquiatría que dirigían él y el psiquiatra Honorio Delgado (1892-1969) un amplio artículo sobre la técnica desarrollada por Moniz (Trelles, 1943); en la misma revista Rocca, Franco, Alayza y Monteagudo (1949) reportan además sus experien- cias. En Chile, Alfonso Asenjo (19061980) y colaboradores (Asenjo, Horvitz, Vergara y Contreras, 1947) dieron a conocer también sus experiencias.

Todavía a mediados del siglo $\mathrm{XX}, \mathrm{y}$ antes de la revolución psicofarmacológica, la lobotomía era considerada como una posibilidad de intervención en el manejo de pacientes psiquiátricos. Uno de los textos de mayor influencia en la psiquiatría latinoamericana, el Curso de psiquiatría, del ya mencionado Honorio Delgado, señalaba expresamente aún en la quinta edición $(1953,1969)$, que en el tratamiento de neurosis, reacciones y desarrollos psíquicos anormales, la leucotomía es el último recurso "para los casos resistentes y graves", dado que "puede librar al paciente de la mortificación de los síntomas o por lo menos mitigarla" (p. 403). Asimismo, que "la leucotomía en los oligofrénicos eréticos da a menudo buenos resultados en lo que respecta a la conducta turbulenta" (p. 355).

El interés por la lobotomía de Pascual del Roncal probablemente guardaba relación con su participación en el movimiento eugenésico en México, pues ella era - como lo destacaba Delgado- una forma de controlar muchos de los "desarrollos psíquicos anormales".

Señalemos, por último, que estos intereses de Pascual del Roncal no constituyen en modo alguno el aspecto más importante de su legado. Son sus trabajos sobre la neuropsiquiatría infantil y el psicodiagnóstico de Rorschach, así como las traducciones que él empren- 
diera, las que hacen de él un protagonista del desarrollo de la psicología en América Latina.

\section{REFERENCIAS}

$A B C$. (17 de febrero de 1936). Concurso de premios de la Sociedad Española de Higiene. $A B C$, edición de la tarde, p. 27.

Álvarez Peláez, R., y Huertas GarcíaAlejo, R. (1987). ¿Criminales o locos? Dos peritajes psiquiátricos del Dr. Gonzalo R. Lafora. Madrid: Consejo Superior de Investigaciones Científicas - Centro de Estudios Históricos, Departamento de Historia de la Ciencia.

Amo, J., y Shelby, Ch. (1950). The printed work of the Spanish intellectuals in the Americas 1936-1945/La obra impresa de los intelectuales españoles en América, 1936-1945. Stanford CA: Stanford University Press.

Asenjo, A., Horvitz, I., Vergara, A., y Contreras, M. (1947). La lobotomía prefrontal como tratamiento de algunas psicosis. Revista Chilena de Neuropsiquiatría, 1 [reimpreso en Revista Chilena de Neuropsiquiatría, 2011, 49(3), 225-240].

Barona, J. L. (2003). Ciencia, salud pública y exilio (España, 1875-1939). Valencia: Seminari D'Estudis sobre la Ciencia.

Boring, E. G. (1950). A history of experimental psychology. Nueva York: Appleton-Century-Crofts.
Boring, E. G. (1988). Historia de la psicología experimental [traducción de Rubén Ardila]. México D. F.: Trillas.

Brosco, J. P., Mattingly, M., y Sanders, M. L. (2006). Impact of specific medical interventions on reducing the prevalence of mental retardation. Archives of Pediatrics \& Adolescent Medicine, 160, 302-309.

Capetillo Hernández, J. (2010). Freud en México. Una revisión panorámica. La Palabra y el Hombre, 44-50.

Cerón González, C. (1996). Luis Valenciano, psiquiatra. Murcia: Universidad de Murcia - Servicio de Publicaciones.

Colotla, V., y Urra, M. (2006). Semblanzas biográficas de los fundadores de la Sociedad Interamericana de Psicología. Revista Interamericana de Psicología, 40(3), 377-384.

Delgado, H. (1953). Curso de psiquiatría. Lima: Imprenta Santa María.

Delgado, H. (1969). Curso de psiquiatría. (5. ${ }^{a}$ ed.). Barcelona: Científico-Médica.

Derbez, J. (1981). Fromm en México. En S. Millán y S. Gojman de Millán (Eds.), Erich Fromm y el psicoanálisis humanista (pp. 27-53). México, D. F.: Siglo XXI Editores.

El-Hay, J. (2007). The lobotomist. New York: Wiley.

Endara, J. (1964). Test de Rorschach: Evolución y estado actual. (2. ${ }^{\mathrm{a}} \mathrm{ed}$.). Quito: Casa de la Cultura Ecuatoriana/Barcelona: Científico-Médica. 
Frolov, Y. P. (1965). La actividad cerebral. Estado actual de la teoría de Pavlov. Buenos Aires: Psique.

Gaceta Médica de México. (31 de diciembre de 1948). Sociedad Mexicana de Eugenesia, 5-6, 448.

González Duro, E. (2009). Guerra civil: una psiquiatría para la represión. En I. Márquez Alonso, A. Fernández Liria y P. Pérez-Sales (Coords.), Violencia y salud mental. Salud mental y violencias institucional, estructural, social y colectiva (pp. 45-60). Madrid: Asociación Española de Neuropsiquiatría.

Gordin, M. (2012). Translating textbooks: Russian, German and the language of chemistry. ISIS: A Journal of the History of Science, 103, 88-98.

Gracia, J. (2010). A la intemperie. Exilio y cultura en España. Barcelona: Anagrama.

Guevara Oropeza, M. (1943). Consideraciones sobre la lobotomía prefrontal, como tratamiento en psiquiatría. Gaceta Médica de México, 72(6), 480-494.

Hernández Rojas, G. (2007). Una reflexión crítica sobre el devenir de la psicología de la educación en México. Perfiles Educativos, XXIX(117), 7-40.

Jelliffe, S. E. (1929). Técnica del psicoanálisis. [Traducción de Honorio Delgado y Paul Wilson]. Madrid: Biblioteca Nueva.
Kevles, D. J. (1995). In the name of eugenics. Genetics and the use of human heredity. Londres-Cambridge, Mass.: Harvard University Press.

Kinsey, A. C., Pomeroy, W. B., y Martin, C. E. (1948). Sexual behavior in the human male. Filadelfia: Saunders.

Kinsey, A. C., Pomeroy, W. B., y Martin, C. E. (1949). Conducta sexual del varón. [Traducción de Federico Pascual del Roncal]. México, D. F.: Editorial Interamericana.

Lee-Jahnke, H. (2005). Teaching medical translation: an easy job? Panace@, 6(20), 81-84.

Lemkau, P. V. (1949). Mental hygiene in public health. New York: McGraw-Hill.

Lemkau, P. V. (1953). Higiene mental. [Traducción de Federico Pascual del Roncal]. México, D. F.: Fondo de Cultura Económica.

León, R. (1981). El Primer Congreso Latinoamericano de Psicología (1950): un evento olvidado. Revista Latinoamericana de Psicología, 13(3), 345-359.

León, R. (2012). F. Oliver Brachfeld y Werner Wolff: dos figuras en los inicios de la Sociedad Interamericana de Psicología. Revista Interamericana de Psicología, 46, 35-42.

León, R., y Kagelmann, H. J. (1992). Zwei Emigranten in Südamerika: der Briefwechsel zwischen Walter Blu- 
menfeld und Emilio Mira y López, Psychologie und Geschichte, 3, 65-76.

León Matamoros, M. G. (2006). De la migración al exilio. Éxodo español a México. Tesis para optar el grado de licenciado en Historia. Benemérita Universidad Autónoma de Puebla, Facultad de Filosofía y Letras. Puebla, México.

López, M. I., y León, N. A. (2001). Historia y orientación del tratamiento psicoanalítico de niños y adolescentes. En Salles Manuel, M. (Coord.), Manual de terapias psicoanalíticas en niños y adolescentes (pp. 19-60). México, D. F.: Plaza y Valdés.

López Albo, W.(1945). Fisiopatología de la imagen somática. Conocimiento corporal normal y patológico. Ciencia. Revista Hispano-Americana de Ciencias Puras y Aplicadas, 6 (4), 145-155. [Recopilación de F. Pascual del Roncal].

López Sánchez, J. M. (enero-febrero de 2009). El Ateneo Español de México y el exilio intelectual republicano. Arbor. Ciencia, Pensamiento y Cultura, 185(735), 41-55.

Marco Igual, M. (2010). Los médicos republicanos españoles en la Unión Soviética. Barcelona: Flor del Viento Ediciones.

Masiero, A. L. (2003). A lobotomía e a leucotomía nos manicomios brasileiros. História, Ciencias, Saude Manguinhos, 10(2), 549-572.
Millán, C., y Bartrina, F. (Eds.). (2013). The Routledge Handbook of Translation Studies. Oxford-New York: Routledge.

Millán, S., y Gojman, S. (2000). The legacy of Fromm in Mexico. International Forum of Psychoanalysis, 9, 207-215.

Mira y López, E. (4 de julio de 1941). Carta a Walter Blumenfeld, desde Buenos Aires.

Montgomery, S. L. (2000). Science in translation: movements of knowledge through cultures and time. Chicago: University of Chicago Press.

Montgomery, S. L. (2009). English and science: realities and issues for translation in the age of an expanding lingua franca. The Journal of Specialized Translation, issue 11, enero, 6-16.

Obrador, S., Pascual del Roncal, F., y Falcón, G. (1943). Un caso de quiste ventricular congénito del lóbulo temporal izquierdo. Archivos de Neurología y Psiquiatría de México, 5, 390-391.

Olohan, M., y Salama-Carr, M. (2011). Translating science. The Translator, 17(2), 179-188.

Páramo Ortega, R. (1992). Freud in Mexiko. Zur Geschichte der Psychoanalyse in Mexiko. Munich: Quintessenz.

Pascual del Roncal, F. (1939a). El fósforo sanguíneo en la epilepsia. Archivos de Neurología y Psiquiatría de México, 2(3). 
Pascual del Roncal, F. (1939b). Blood phosphorus in mental disease. Journal of Nervous and Mental Diseases, 94(2), 133-146.

Pascual del Roncal, F. (1940a). Organización de los servicios anexos de un Tribunal de Menores. Criminalia, 6, 265-284.

Pascual del Roncal, F. (1940b). Manual de neuropsiquiatría infantil. México, D. F.: Casa de España.

Pascual del Roncal, F. (1941a). Las clínicas de conducta y su importancia en la prevención de la delincuencia juvenil. Criminalia, 7, 409-416.

Pascual del Roncal, F. (1941b). Fundamentos biológicos de la herencia. Eugenesia, 25(2), 4-13.

Pascual del Roncal, F. (1944). Las nuevas orientaciones del psicoanálisis. Cuadernos Americanos, 1(13), 117-124.

Pascual del Roncal, F. (1945). Dos casos de demencia precosísima. Archivos de Neurología y Psiquiatría de México, $8,1$.

Pascual del Roncal, F. (1947a). Cuarenta casos de lobotomía prefrontal. Revista Médica. Ateneo Ramón y Cajal, 2, 279-291.

Pascual del Roncal, F. (1947b). El psicodiagnóstico de Rorschach en los lobotomizados, Revista Médica. Ateneo Ramón y Cajal, 2, 511-513.

Pascual del Roncal, F. (1947c). Recensión de Los engramas de la psiquiatría, de J. M. Nielsen y G.
N. Thompson. Ciencia. Revista Hispano-Americana de Ciencias Puras y Aplicadas, 6-9, 223.

Pascual del Roncal, F. (1949). Teoría y práctica del psicodiagnóstico de Roschach. México, D. F.: The University Society Mexicana.

Pascual del Roncal, F. (1951). Acción del propionato de testosterona (perandren) sobre el estado psíquico de los ancianos. Revista de Medicina y Ciencias Afines, 9, 654-685.

Pascual del Roncal, F. (1953). La psiquiatría en el teatro. Lotería. Órgano de la Lotería Nacional de Beneficencia, 143, 18-20.

Pascual del Roncal, F. (1954). El Rorschach en criminología. Humanitas. Revista de la Universidad Nacional de Tucumán, 3, 295-304.

Petrou, G. (2006). Translation studies and the history of science: the Greek textbooks of the $18^{\text {th }}$ century. Science \& Education, 15(7-8), 823-840.

Pimenta, A. M. (1936) 'Leucotomia cerebral (a psico-cirurgia de Egas Moniz)'. Arquivos da Assistencia Geral a Psicopatas do Estado de São Paulo, I, 1, 259-266.

Quintana, J., Rosa, A., Huertas, J. A., y Blanco, F. (Eds.). (1997). La incorporación de la psicología científica a la cultura española. Siete décadas de traducciones (1868-1936). Alcalá de Henares: Universidad Autónoma de Madrid. 
Rocca, E. D., Franco, J., Alayza, F., y Monteagudo, E. (1949). Estado actual de la leucotomía. Revista de Neuro-psiquiatría, 12, 328-339.

Rupke, N. (2000). Translation studies in the history of science: the example of Vestiges. The British Journal for the History of Science, 33(2), 209-222.

Schilder, P. (1945). Tratado de psicoterapia. [Traducción de Emilio Mira y López]. Buenos Aires: Paidós.

Shuping, R. (2013). Translation and rewriting. International Journal of Humanities and Social Sciences, 3(18), 55-59.

Skinner, B. F. (1957). Verbal behavior. Nueva Jersey: Prentice Hall.

Skinner, B. F. (1981). Conducta verbal. [Traducción de Rubén Ardila]. México, D. F.: Trillas.

Sluchevski, I. F. (1960). Psiquiatría. [Traducción del ruso de Florencio Villa Landa y Manuel de la Loma]. México, D. F.: Grijalbo.

Smirnov, A. A., (1960). Psicología. México, D. F.: Grijalbo.
Suárez y López Guazo, L. (2000). La antropología criminal y su influencia en el campo de la salud mental en México. $L L U L L, 23,689-709$.

Trelles, J. O. (1943). La leucotomía prefrontal de Egas Moniz. Revista de Neuro-psiquiatría, 6, 328-363.

Varela Fregoso, P. J., y Vásquez Lorenzana, A. D. (2014). El Instituto Mexicano de Psicoanálisis. A 50 años de su fundación por Erich Fromm. Consciencia y Diálogo, 4(4), 169-176.

Wolff, W. (1947). What is psychology. A basic survey. Nueva York: Grune $\&$ Stratton.

Wolff, W. (1950). Introducción a la psicopatología. México, D. F.: Fondo de Cultura Económica.

Wolff, W. (1953). Introducción a la psicología. [Traducción de Federico Pascual del Roncal]. México, D. F.: Fondo de Cultura Económica.

Wolff, W. (1956). Introducción a la psicopatología. [Traducción de Federico Pascual del Roncal]. México, D. F.: Fondo de Cultura Económica. 
